\title{
Alien Intracellular Calcium Chelators Attenuate Neurotransmitter Release at the Squid Giant Synapse
}

\author{
E. M. Adler, ${ }^{1,2}$ George J. Augustine, ${ }^{2,3}$ Steven N. Duffy, ${ }^{1,2, a}$ and Milton P. Charlton ${ }^{1,2}$ \\ 'Physiology Department, University of Toronto, Toronto, Ontario, Canada M5S 1A8, ${ }^{2}$ Marine Biological Laboratory, Woods \\ Hole, Massachusetts 02543, and ${ }^{3}$ Section of Neurobiology, Department of Biological Sciences, University of Southern \\ California, Los Angeles, California 90089-0371
}

\begin{abstract}
A number of calcium buffers were examined for their ability to reduce evoked transmitter release when injected into the presynaptic terminal of the squid giant synapse. Injection of EGTA was virtually ineffective at reducing transmitter release, even at estimated intracellular concentrations up to $80 \mathrm{~mm}$. Conversely, the buffer 1,2-bis(2-aminophenoxy)ethane- $N, N, N^{\prime}, N^{\prime}$-tetraacetic acid (BAPTA), which has an equilibrium affinity for calcium similar to that of EGTA at $\mathrm{pH}$ 7.2, produced a substantial reduction in transmitter release when injected presynaptically. This effect of BAPTA was reversible, presumably because the buffer diffused out of the terminal and into uninjected regions of the presynaptic axon. BAPTA derivatives with estimated intracellular calcium dissociation constants $\left(K_{d}\right)$ ranging from 0.18 to $4.9 \mu \mathrm{M}$ were effective at reducing transmitter release at similar estimated concentrations. A BAPTA derivative with an estimated intracellular $K_{d}$ of $31 \mathrm{~mm}$ was less effective. BAPTA did not affect presynaptic action potentials or calcium spikes in ways that could explain its ability to reduce transmitter release. The relative effects of presynaptic injections of BAPTA and derivatives are consistent with the calcium-buffering capability of these compounds if the presynaptic calcium transient that triggers release is hundreds of $\mu \mathrm{M}$ or larger. The superior potency of BAPTA compared to EGTA apparently results from the faster calcium-binding kinetics of BAPTA and suggests that the calcium-binding molecule that triggers release binds calcium in considerably less than $200 \mu \mathrm{sec}$ and is located very close to calcium channels.
\end{abstract}

Many lines of evidence suggest that the release of neurotransmitter is triggered by a brief increase in the intracellular calcium concentration of the presynaptic nerve terminal (Katz, 1969). One prediction of this calcium hypothesis of neurotransmitter

\footnotetext{
Received Sept. 4, 1990; revised Dec. 19, 1990; accepted Dec. 27, 1990.

We thank M. Hines for discussions on diffusion modeling, L. F. Jaffe for extensive discussions on intracellular calcium buffering and especially for suggesting the synthesis of dinitroBAPTA, A. Klip for the use of her fluorescence spectrophotometer, S. J. Smith for helping to assemble the vidco imaging system, and R. Bookman, J. Moore, and P. Pennefather for comments on the manuscript. This work was supported by NIH Grant NS-21624 to G.J.A. and by a Medical Research Council grant to M.P.C. E.M.A. was supported by the Department of Physiology, University of Toronto. S.N.D. was supported by a University of Toronto Open Fellowship.

Correspondence should be addressed to Dr. E. M. Adler, Laboratory of Molecular Neurobiology, Massachusetts General Hospital East, 149 13th Street, Charlestown, MA 02129.

a Present address: Department of Medical Physiology, University of Calgary, Calgary, Alberta, Canada T2N 4N1.

Copyright (C) 1991 Society for Neuroscience $0270-6474 / 91 / 111496-12 \$ 03.00 / 0$
}

release is that an increase in the intracellular calcium-buffering capacity should dampen this transient rise in free-calcium concentration and hence attenuate transmitter release (see Nachsen and Drapeau, 1982). However, Adams et al. (1985) have reported that injection of the calcium chelator EGTA into the presynaptic nerve terminal of the squid giant synapse did not reduce evoked transmitter release. This observation could be interpreted as contradicting the calcium hypothesis. Indeed, similar experiments in rat mast cells, another system in which calcium has been considered essential to secretion, have led to the suggestion that an increase in intracellular calcium is not required to trigger histamine release (Neher, 1988).

Alternatively, the lack of EGTA effect on transmitter release could instead relate to the specifics of EGTA-calcium binding compared to the requirements of the endogenous calcium-binding molcculc or molcculcs that trigger ncurotransmitter relcase (Thomas, 1982; Adams et al., 1985; Marty and Neher, 1985; Neher, 1986). This study was undertaken in order to investigate the effects on transmitter release of a series of buffers with a wide range of affinities and binding rates for calcium.

We find that, while EGTA has little effect on evoked neurotransmitter release, buffers with similar equilibrium calcium affinities but faster binding kinetics produce a pronounced altenuation of release. This argues that the lack of effect of EGTA results from its slow calcium-binding kinetics rather than from an inadequacy in the calcium hypothesis of neurotransmitter release. The relative efficacy of several calcium buffers in attenuating evoked release suggests that the peak free-calcium concentration attained during the transient may be on the order of hundreds of $\mu \mathrm{M}$ or greater and that the calcium-binding molecule or molecules involved in triggering neurotransmitter release bind calcium very rapidly and are located very close to the presynaptic calcium channels.

Preliminary reports of some of these results have appeared previously (Adler et al., 1988a,b).

\section{Materials and Methods}

Synaptic transmission was investigated at the most distal giant synapse of stellate ganglia from small $(5-15 \mathrm{~cm})$ Loligo pealei or $L$. opalescens. Isolation and maintenance of the ganglia were as previously described (Charlton and Bittner, 1978a; Augustine and Eckert, 1984).

At $50-$ or $60-\mathrm{sec}$ intervals, the presynaptic neuron was stimulated intracellularly by a current pulse passed through a microelectrode filled with either $3 \mathrm{M} \mathrm{KCl}$ or calcium chelator (in experiments where two different buffers were injected sequentially into the same nerve terminal). In this case, the electrode not currently being used for buffer iontophoresis was used to stimulate the nerve terminal. The presynaptic action potential was recorded at the nerve terminal with the iontophoresis electrode. 
Transmitter release was assayed by rccording the electrical response of the postsynaptic neuron to presynaptic stimulation. Usually, transmitter release was quantified by measuring $d V / d t$ of the postsynaptic potential (PSP) at a given criterion voltage above resting membrane potential; this allowed measurement of responses even when PSPs exceeded threshold for initiation of a postsynaptic action potential. In any given experiment, this criterion voltage was established as that voltage at which maximum $d V / d t$ occurred for the smallest recorded PSP. In some experiments, postsynaptic current (PSC) was measured using a two-microelectrode voltage clamp (Augustine et al., 1985). In this case, PSCs were quantified by measuring their peak amplitude. In most experiments, electrophysiological signals were digitized, stored, and analyzed with a PDP-11/23 computer system. In a few experiments, signals were measured on a storage oscilloscope screen.

Measurements were made on isolated ganglia superfused with artificial seawater consisting of (in $\mathrm{mM}$ ) $\mathrm{NaCl}, 466 ; \mathrm{MgCl}_{2}, 54 ; \mathrm{CaCl}_{2}, 11$; $\mathrm{KCl}, 10$; $\mathrm{NaHCO}_{3}, 3$; and HEPES, $10\left(\mathrm{pH}, 7.2\right.$ at $12-16^{\circ} \mathrm{C}$; oxygenated with $99.5 \% \mathrm{O}_{2}, 0.5 \% \mathrm{CO}_{2}$ ). In three experiments with $5,5^{\prime}$-dinitro-1, 2-bis(2-aminophenoxy)ethane- $N, N, N^{\prime}, N^{\prime}$-tetraacetic acid (dinitroBAPTA), where transmission was exceptionally robust, calcium concentration was reduced to $5.5 \mathrm{~mm}$ in order to reduce transmitter release. In one experiment with EGTA, $10 \mu \mathrm{M}$ nimodipine was added to the bath in order to inhibit ganglionic contraction (Sanchez et al., 1990).

\section{Calcium chelator microinjection}

The following calcium buffers were evaluated for effects on synaptic transmission: BAPTA, 5,5'-dimethylBAPTA, 5,5'-dibromoBAPTA, 5,5'dinitroBAPTA (all from Molecular Probes, Eugene, OR), and ethyleneglycol-bis-( $\beta$-aminoethyl ether)- $N, N^{\prime}$-tetraacetic acid (EGTA) (Sigma). DinitroBAPTA was custom synthesized by Molecular Probes (on the suggestion of L. F. Jaffe). The EGTA solution was adjusted to $\mathrm{pH} 7$ with $\mathrm{KOH}$.

\section{Iontophoretic parameters}

Calcium buffers were injected iontophoretically from glass microelectrodes filled with 0.25 or $0.50 \mathrm{M}$ buffer. Currents of $100-200 \mathrm{nA}$ were passed between the buffer-filled electrode and ground for 30-sec sessions repeated at 1 -min intervals between successive nerve stimulations. This hyperpolarized the presynaptic membrane potential by $10-45 \mathrm{mV}$ for the duration of the current application. Five to $20 \mathrm{sec}$ elapsed between cessation of iontophoresis and nerve stimulation to allow membrane repolarization. As has previously been reported (Miledi and Slater, 1966), similar hyperpolarizations produced by passing current with $3 \mathrm{M} \mathrm{KCl}-$ containing microelectrodes had no sustained effect on transmitter release.

\section{Buffer concentration}

To compare the actions of different buffers and predict their probable effects on calcium concentration requires an estimate of the intraterminal buffer concentration. This relies on two parameters: the amount of buffer iontophoretically injected and the intracellular volume through which it is distributed.

Transference number. The amount of buffer iontophoresed into the terminal can be calculated from the current passed, the valence of the injected species, and the transference number:

$$
n=\mathrm{I} t \mathrm{~T}_{\mathrm{b}} / F z
$$

in which $n$ is moles injected, $\mathrm{I}$ is number of amperes, $t$ is time in seconds, $T_{b}$ is the transference number (i.e., the fraction of total current carried by the species of interest), $F$ is the faraday, and $z$ is the valence. It should be noted that, while the BAPTA-family buffers are tetraanions at neutral $\mathrm{pH}$, EGTA still has two associated protons and is a dianion. Therefore, for the same transference number and total coulombs passed, twice as much EGTA will be iontophoretically injected. The only experimental unknown for the buffer iontophoreses is the transference number. This value was determined for BAPTA as follows:

Microelectrodes containing 0.25 or $0.50 \mathrm{~m}$ BAPTA were used to pass current $(100$ or $200 \mathrm{nA})$ into $200-\mu \mathrm{l}$ volumes of saline, similar in cationic composition to squid cytoplasm (in mM): $\mathrm{KCl}, 380 ; \mathrm{NaCl}, 50 ; \mathrm{MgCl}_{2}$, 10; HEPES, 10; and EGTA, 5 (to maintain the iontophoresed chelator in the calcium-free form); $\mathrm{pH} 7.2$. A 10-sec-on, 10-sec-off current cycle was used to mimic the interrupted iontophoretic parameters used experimentally. In order to obtain detectable BAPTA concentrations, it was necessary to continue iontophoresis for 3-6 hr. Evaporative weight loss was measured, and volume was accordingly restored.

BAPTA concentration was measured fluorometrically on a Perkin Elmer 650-40 fluorescence spectrophotometer. Excitation was at 254 $\mathrm{nm}$ (5-nm slit width), which corresponds to the peak absorbance for the calcium-free form of BAPTA (Tsien, 1980), and emission was measured at $375 \mathrm{~nm}$ (15-nm slit width), which was the peak of emission under our conditions. As has previously been reported (Harrison and Bers, 1987), BAPTA (Molecular Probes, lot 6D) contained about 20\% water by weight. (Following the procedure of Harrison and Bers, three samples were dried to constant weight at $150^{\circ} \mathrm{C}$. Weight loss ranged from $20 \%$ to $21.4 \%$.) Therefore, an additional $25 \%$ by weight was included in the BAPTA fluorometry standards.

The transference number for BAPTA ranged from 0.06 to 0.22 under various conditions of iontophoretic current and buffer concentration (0.5 M BAPTA at $100 \mathrm{nA:} \mathrm{T}_{\mathrm{b}}=0.10 \pm 0.03, n=7 ; 0.25 \mathrm{M}$ BAPTA at $100 \mathrm{nA}: \mathrm{T}_{b}=0.22 \pm 0.05, n=6 ; 0.5 \mathrm{~m}$ BAPTA at $200 \mathrm{nA}: \mathrm{T}_{b}=0.06$ $\pm 0.01, n=7$; mean \pm SEM). It was assumed that EGTA and all of the other compounds, which are closely related structurally, have transference numbers in the same range.

Presynaptic terminal volume. Nerve terminal dimensions (length, width at distal tip, width at base, the proximal junction with axon) were estimated with an eyepiece micrometer, and terminal volume was calculated as a cylinder or truncated cone. A "typical" nerve terminal was $750 \mu \mathrm{m}$ long, $50 \mu \mathrm{m}$ wide at the tip, and $80 \mu \mathrm{m}$ wide at the base. The site of buffer injection along the nerve terminal was not standardized from experiment to experiment, but usually this was about one-third to one-half of the distance from the base to the tip. For the purposes of calculating buffer concentration at the end of the loading period, the assumption was made that, during loading, buffer distributed throughout the nerve terminal but had not yet begun to diffuse down the presynaptic axon. Visual observation of the nerve terminal during loading of dinitroBAPTA, which is colored yellow, supported this approximation (see below). Buffer concentration at the end of the loading period was calculated by dividing the total number of moles injected by the volume of the nerve terminal.

Using the above assumptions, buffer concentration in the nerve terminal at the end of a single 30-sec iontophoretic session was estimated to be on the order of hundreds of $\mu \mathrm{M}$. Usually, loading was stopped after 10 or 12 iontophoretic sessions (i.e., mM concentrations).

\section{Buffer distribution}

The diffusion coefficient, $D$, expected for molecules of this size is about $2 \times 10^{-6} \mathrm{~cm}^{2} \mathrm{sec}^{-1}$ in free solution (Neher, 1986). If buffer diffusion in cells is severely retarded by binding to intracellular constituents (Baylor and Hollingworth, 1988), the 10-12-min loading period might be insufficient to allow buffer distribution throughout the nerve terminal. In this case, steep intraterminal buffer concentration gradients could exist, making it difficult to calculate a meaningful average terminal buffer concentration (though comparison of the relative effects of different buffers should still be valid). The related questions of buffer diffusion coefficient and intraterminal homogeneity of concentration were addressed by optically monitoring the distribution of the colored BAPTA derivative dinitroBAPTA in the presynaptic nerve terminal.

Video microscopic examination of buffer diffusion. To view the spread of dinitroBAPTA, preparations were examined in an upright microscope (Zeiss Standard) equipped with a $6.3 \times$ Neofluar objective and stagemounted hydraulic manipulators (Narishige WR-88). Presynaptic terminals were impaled with dinitroBAPTA-filled microelectrodes, and dinitroBAPTA was injected iontophoretically, as described above. Preparations were illuminated with a tungsten lamp, and the resultant light was filtered to an effective bandwidth of approximately $360-500 \mathrm{~nm}$, the range of maximum absorbance of dinitroBAPTA (Pethig et al., 1989). Although the optical absorbance of dinitroBAPTA is calcium sensitive in this wavelength range, calcium-dependent changes in absorbance occur at calcium concentrations much higher than those expected in presynaptic terminals at rest (see Table 1; Pethig et al., 1989). Thus, changes in light transmission produced by dinitroBAPTA injection into the presynaptic terminal likely reflected changes in dinitroBAPTA concentration. Images of synapses were obtained during dinitroBAPTA injection by using an SIT video camera (Cohu 5000) attached to the microscope. These images were stored at a rate of $0.5-$ $2 \mathrm{sec}^{-1}$ on an analog optical disk recorder (Panasonic TQ-2026F) operating in a time-lapse mode and were digitized off line with a Matrox MVP-AT image processor. Digitized images were manipulated with 
software written by S. J. Smith and with IMAGE-PRo II (Media Cybernetics, Silver Spring, MD).

Determination of D. DinitroBAPTA spread rapidly within the nerve terminal after being injected (Fig. 1). This was quantified by measuring the spatial profile of light transmission at various times before and after buffer injection. The difference between images obtained before and after buffer injection was taken as a measure of dye-dependent transmission changes. Because the thickness of the nerve terminal (the effective path length for these measurements) varies along the length of the synapse, it was necessary to normalize the measured changes in light transmission in order to estimate the relative changes in buffer concentration along the nerve terminal. This was done by measuring the profile of changes in light transmission remaining $20 \mathrm{~min}$ after buffer injection, by which time it was assumed that the concentration of buffer within the nerve terminal should be spatially uniform, in order to obtain a signal proportional to path length, and dividing this into the transmission change profiles obtained at earlier times. The resulting normalized profiles were steep at early times and gradually flattened at later times after buffer injection (Fig. $2 A$ ).

The spatial profile should be described by the equation for diffusion from a plane into an infinite cylinder of unit cross section. In this case, buffer concentration, $C_{(x, t)}$, is given by

$$
C_{(x, t)}=\frac{N_{(0)}}{2 \mathrm{~F}(\pi D t)^{1 / 2}} e^{-x^{2} / 4 D t},
$$

where $x$ is the distance along the axon at any time, $t$, and $N_{10}$ is the total number of molecules of buffer injected into the terminal. The data of Figure $2 A$ were fit to this equation by a statistical curve-fitting program (STATGRAPHICS, STSC Corporation; nonlinear regression using the Marquardt algorithm), which determined the best value for the diffusion coefficient, $D$, and a scaling factor, $\mathrm{F}$, necessary to correct for the actual cross-sectional area (Fig. $2 B$ ). In this experiment, the average value of $D$ determined at six intervals from 0 to $160 \mathrm{sec}$ after injection was 1.66 $\pm 0.11 \times 10^{-6} \mathrm{~cm}^{2} \mathrm{sec}^{-1}$. When values determined in two additional experiments using longer iontophoretic injections were included, the average value of $D$ was $1.71 \pm 0.07 \times 10^{-6} \mathrm{~cm}^{2} \mathrm{sec}^{-1}(n=10)$

A similar $D$ has been found for a closely related compound, fura-2, in lamprey axons (Strautman et al., 1990), and it also appears that fura-2 diffuses at similar rates in chromaffin cells (Pusch and Neher, 1988). It seems unlikely that there would be gross differences in $D$ for the other buffers we used because they are closely related chemically and have similar molecular weights (Tsien, 1989). The values of $D$ obtained for dinitroBAPTA and fura-2 are similar to the values expected for these molecules in free solution (Neher, 1986) and suggest little binding to intracellular constituents. The retardation of fura- 2 diffusion seen in skeletal muscle cells (Baylor and Hollingworth, 1988) may be due to the relatively large amounts of cytoskeletal proteins present in these cells.

Determination of intraterminal chelator concentration profiles. The flattened profile of the spatial scan taken $640 \mathrm{sec}$ after starting a brief injection shows little evidence of dinitroBAPTA concentration gradients within the nerve terminal over a range of about $800 \mu \mathrm{m}$ (Fig. $2 A$ ). The question of intraterminal buffer homogeneity was examined further using the multiple-pulse injection protocol that was used in the experiments on transmitter release.

DinitroBAPTA was iontophoresed for $30 \mathrm{sec}$ at $100 \mathrm{nA} 10$ times at 1-min intervals. During the 10-min iontophoretic loading period, and for 10 min thereafter, optical transmission was monitored at three points: at the injection site, at the tip of the nerve terminal $(\sim 350 \mu \mathrm{m}$ from injection site), and in the preterminal axon ( $\sim 620 \mu \mathrm{m}$ from the injection site; $\sim 130 \mu \mathrm{m}$ from the base of the terminal; Fig. $3 A$ ). Normalized optical transmission changes showed steep intraterminal concentration gradients during the period of iontophoresis, as evident from the differences between traces a and $\mathrm{b}$ in Figure $3 B$, but relatively small gradients were apparent after cessation. The slow rise of trace $c$ indicates that little buffer had escaped the terminal $5 \mathrm{~min}$ after initiation of iontophoresis, though some buffer was clearly detectable beyond the terminal by the end of the iontophoretic session.

These observations suggest that the approximations used to determine buffer concentration are reasonable. While buffer concentration gradients do exist during the injection period, they diminish considerably by the end of the loading period. Some of the chelator does appear to escape from the nerve terminal during iontophoresis, as indicated by the change in light transmission measured in the preterminal axon, but

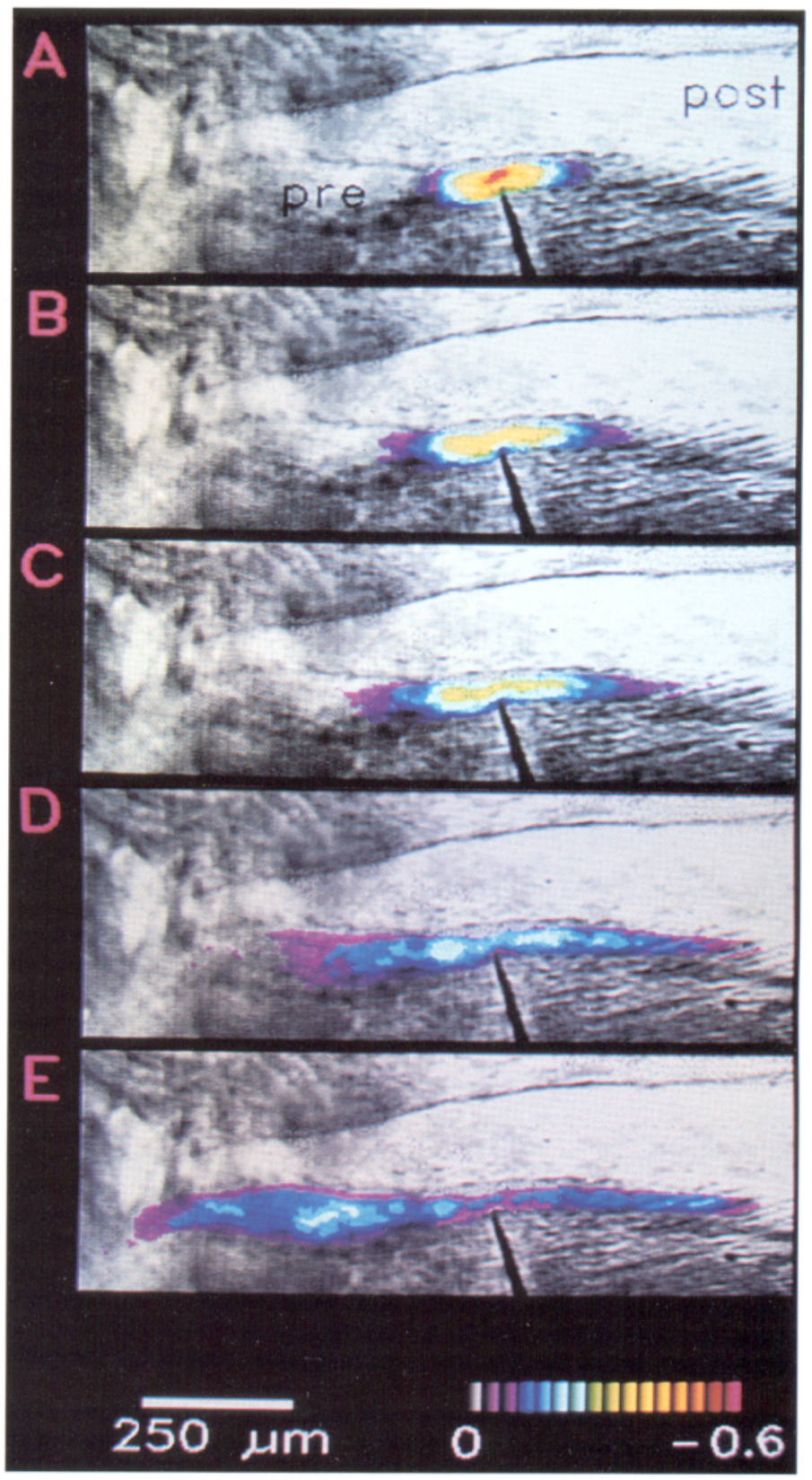

Figure 1. Digital imaging of dinitroBAPTA diffusion in the presynaptic nerve terminal. DinitroBAPTA was iontophoretically injected for $10 \mathrm{sec}$ at $1-\mu \mathrm{A}$ current. Successive images taken at $0,10,20,80,160$ and $640 \mathrm{sec}$ after stopping the injection are shown in $A-E$. These images indicate the decreases in light transmission caused by dinitroBAPTA (relative to maximum light transmission in the image field) and are encoded in the pseudocolor scale shown at the bottom of the figure. The changes in light transmission are overlayed on a bright-field image of the synapse to indicate the position of the presynaptic (pre) and postsynaptic (post) elements.

this appears to be a relatively minor fraction of the entire terminal buffer content.

\section{Estimation of dinitroBAPTA concentration}

We have used the changes in optical transmission produced by dinitroBAPTA injection to provide an independent estimate of the concentration of dinitroBAPTA in the nerve terminal. By Beer's law,

[dinitroBAPTA] $=a / E l$, 

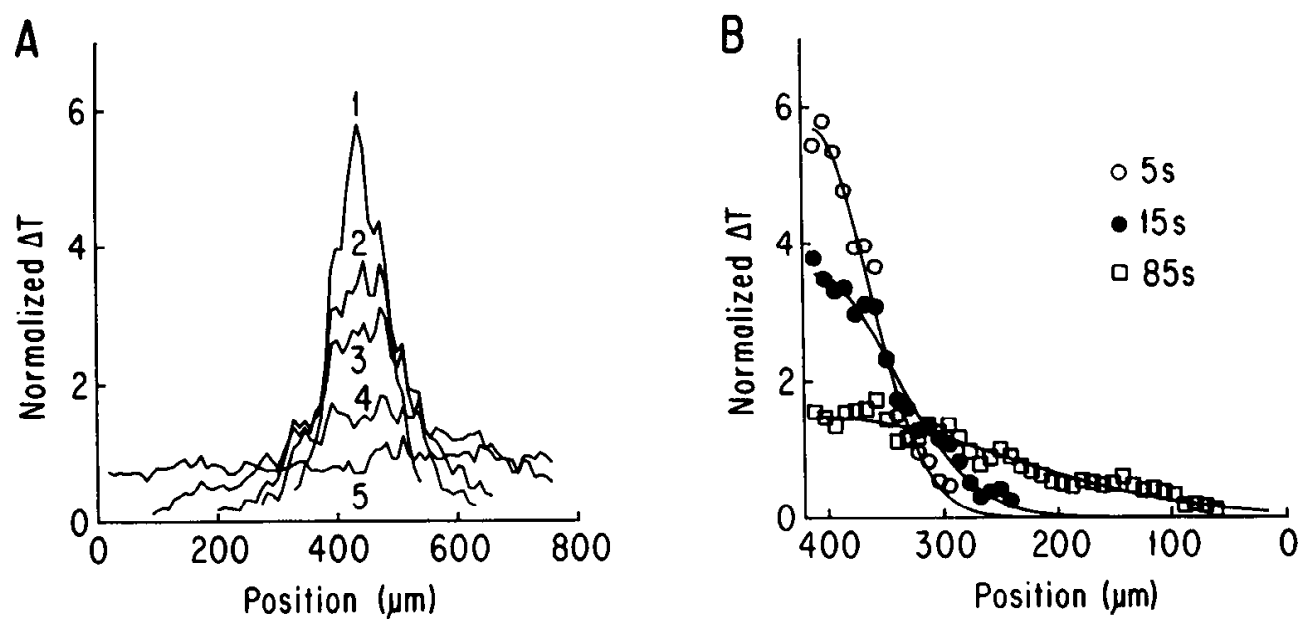

Figure 2. $A$, Profiles of spatial and temporal changes in relative light transmission $(\Delta T)$ during the dinitroBAPTA injection shown in Figure 1. The changes in transmission caused by dinitroBAPTA were normalized by dividing by the transmission measured $20 \mathrm{~min}$ after injection. Time points $0,10,20$, 80 , and $640 \mathrm{sec}$ after stopping iontophoresis are indicated by curves $1,2,3$, 4 , and 5 , respectively. $B$, The left-hand limbs of curves 1, 2, and 4 from $A$ (symbols) have been superimposed on the best-fit solutions of Equation 2 (continunus curves) for $t=5,15$, and $85 \mathrm{sec}$. These times are calculated relative to the midpoint of the 10-sec-long injection. Equation 2 optimally fit the data when the diffusion coefficient for dinitroBAPTA was set at $2.16,1.56$, and $1.50 \times 10^{-6} \mathrm{~cm}^{2} \mathrm{sec}^{-1}$. where $a$ is absorbance, $l$ is path length, and $E$ is the extinction coefficient of dinitroBAPTA. For the experiment shown in Figure 3, the change in light transmission caused by dinitroBAPTA injection ( $\Delta$ trans) was approximately $50 \%$ when measured at point $b$ in the nerve terminal (see Fig. 3A) $4 \mathrm{~min}$ after loading. From the relationship

$$
10^{-a}=1 / \Delta \text { trans, }
$$

absorbance was calculated to be 0.3 . The average $E$ (measured at 360 $500 \mathrm{~nm}$ in the absence of calcium) for the same lot of dinitroBAPTA used in the injections was $1.4 \times 10^{4} \mathrm{M}^{-1} \mathrm{~cm}^{-1}$. Although the thickness of the nerve terminal, and hence $l$, was not known, it was approximately $100 \mu \mathrm{m}$ and certainly no less than $50 \mu \mathrm{m}$. Thus, the estimated dinitroBAPTA concentration is approximately $2.2 \mathrm{~mm}$ and certainly no more than $4.3 \mathrm{~mm}$. Calculation of the average concentration of dinitroBAPTA using Equation 2, modified for the actual dimensions of the nerve terminal and diffusional loss of buffer after cessation of loading (see below), yielded a value of $2.15 \mathrm{~mm} 4 \mathrm{~min}$ after loading. Given the uncertainty about the thickness of the nerve terminal, and the necessity of using an average value for $E$, the correlation between the two estimates is certainly reasonable and supports the validity of the assumptions used to calculate average buffer concentrations.

A

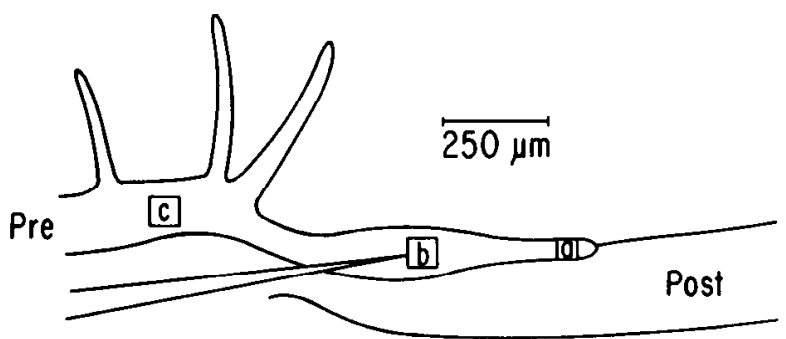

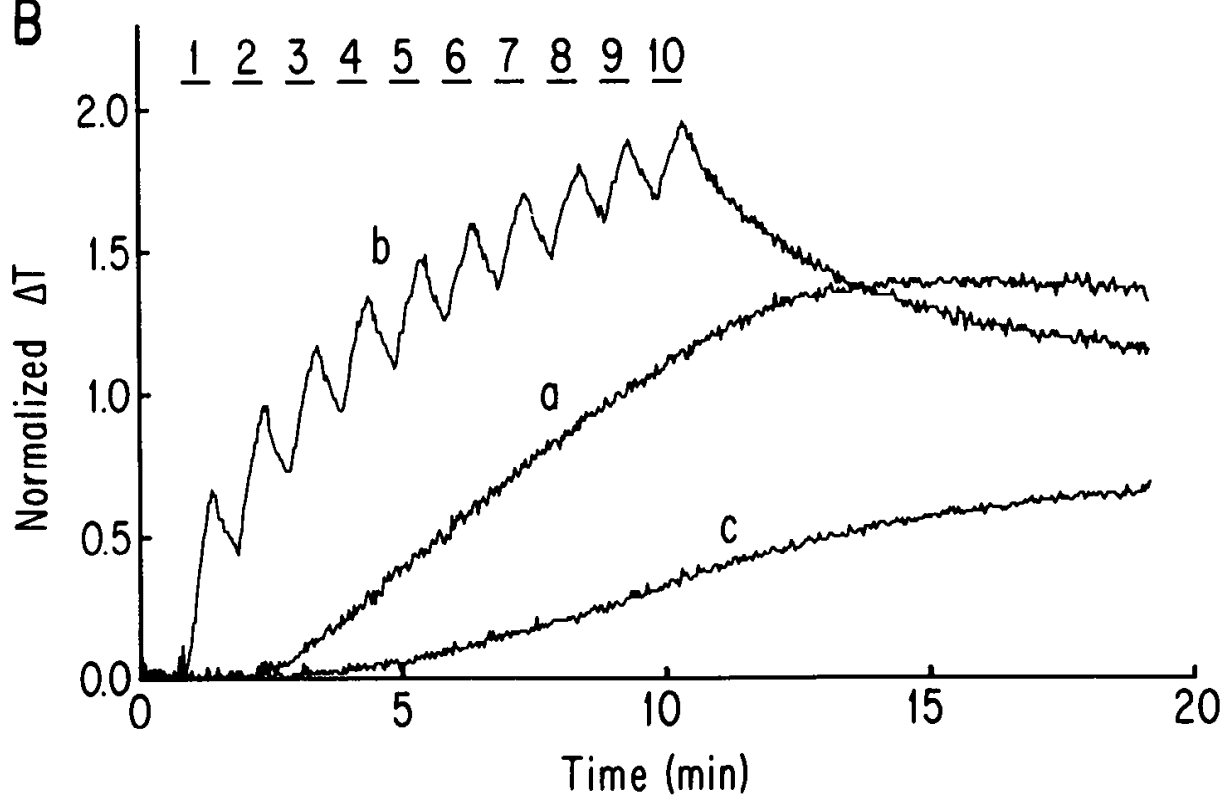

Figure 3. The spatial and temporal profile of light transmission changes caused by multiple injections of dinitroBAPTA. $A$, Diagram of experimental arrangement, showing the location of the injection pipette and the sites of measurement of changes in optical transmission resulting from dinitroBAPTA injection. $B$, Following the same iontophoretic protocol used to examine buffer effects on synaptic transmission, 10 30-sec 100-nA iontophoretic pulses were given at 1-min intervals, indicated by the bars numbered $1-10$. Curve $b$ shows the changes in relative light transmission $(\Delta T)$ at the injection site, curve $a$ at the tip of the terminal, and curve $c$ in the preterminal axon, corresponding to the areas indicated in $A$. All three curves have been normalized for variation in path length by dividing by the changes in optical transmission remaining $40 \mathrm{~min}$ after injection. Pre, presynaptic; Post, postsynaptic. 


\section{Calculation of $K_{d}$}

The $K_{d} \mathrm{~s}$ of the various buffers are affected by both temperature and ionic strength (Harrison and Bers, 1987). Under the present experimental conditions of high ionic strength and low temperature, the calcium affinities of BAPTA-family buffers will be less than the published values measured at $22^{\circ} \mathrm{C}$ in $0.1 \mathrm{M} \mathrm{KCl}, 0.01 \mathrm{M}$ HEPES (Tsien, 1980). Additionally, the $K_{d}$ of EGTA for calcium is markedly influenced by $\mathrm{pH}$ in the physiological range (Thomas, 1982). Because a major purpose of this study was to compare the effects of a series of calcium chelators of varying $K_{d}$, we have calculated $K_{d} \mathrm{~s}$ for the following conditions: ionic strength $=0.453 ; T=287^{\circ} \mathrm{K} ; \mathrm{pH}=7.0-7.3$.

Ionic strength was calculated as ionic equivalence $I=0.5 \Sigma \mathrm{m}|z|$, where $m$ is molal concentration and $z$ is valence (see Smith and Miller, 1985 ) for a solution containing, in $\mathrm{mmol} / \mathrm{kg}, \mathrm{K}^{+}, 362 ; \mathrm{Na}^{+}, 50 ; \mathrm{Mg}^{2+}$, 13; $\mathrm{SO}_{4}{ }^{2-}, 7.5$; and anion-, 453. These values represent the midpoints of the ranges for cation concentration of squid axoplasm given in Gainer et al. (1984). The calculated values are likely to be somewhat inaccurate both bccause of uncertainty as to the exact composition of squid axoplasm, and because the equations used are more accurate for dilute solutions. They should, however, be much closer to the true intracellular $K_{d} s$ than uncorrected values measured under standard conditions of ionic strength and temperature. In any case, this correction should not distort the relationship between the $K_{d} \mathrm{~s}$ of the various buffers.

In order to correct for the change in ionic activity and the increase of $K_{d}$ by high ionic strength, we have used the equations of Smith and Miller (Miller and Smith, 1984; Smith and Miller, 1985), which are derived from the Guggenheim approximation to the Debye-Hückel limiting law (see Davies, 1962, pp. 34-53):

$$
\log K^{\prime}=\log K-\log f^{\prime},
$$

where $K^{\prime}$ is the association constant to be calculated for experimental ionic strength, $K$ is the association constant measured under standard conditions, and $\log f^{\prime}$ is defined by

$$
\log f^{\prime}=2 x y\left[\alpha\left(\frac{I^{1 / 2}}{1+I^{1 / 2}}-\beta I\right)-\alpha\left(\frac{I^{\prime / 2}}{1+I^{\prime / 2}}-\beta I^{\prime}\right)\right]
$$

which corrects the ion activity coefficient for ionic strength. $x$ and $y$ are the charges on cation and anion, respectively; $I$ is ionic strength under standard conditions; $I^{\prime}$ is ionic strength under experimental conditions; $\beta$ is a variable coefficient. Smith and Miller (1985) empirically found that $\beta=0.25$ best described the decrease in ion activity with increasing ionic strength for their solutions. $\alpha$ is calculated from

$$
\alpha=\left(1.8246 \times 10^{6}\right) /(\epsilon T)^{3 / 2},
$$

where $\epsilon$ is the dielectric constant of water and $T$ is the temperature Kelvin. The correction was made for ionic strength of $0.453 \mathrm{M}$ at $295^{\circ} \mathrm{K}$.

Harrison and Bers (1987) found that $K^{\prime}$ calculated for dibromoBAPTA, unlike that for BAPTA and EGTA, was consistently below that measured empirically. The difference amounted to $0.19 \log$ units at $0.304 \mathrm{M}$ (the highest ionic strength they measured). Therefore, this amount has been added to the $K_{d}$ calculated for dibromoBAPTA. Because no information comparing the theoretical to empirical values of $K^{\prime}$ for dimethyl- or dinitroBAPTA is available at present, the theoretical values of these two compounds have been used.

Following Harrison and Bers (1987), the Van't Hoff Isochore was used to correct the $K_{d}$ s for experimental temperature $\left(287^{\circ} \mathrm{K}\right)$ :

$$
\log K^{\prime}=\log K-\Delta H\left[\frac{T-T^{\prime}}{(T)\left(T^{\prime}\right)}\right]\left[\frac{1}{(2.303 R)}\right]
$$

The $\Delta H$ values empirically determined by Harrison and Bers (1987) for EGTA $(16.6 \mathrm{~kJ} / \mathrm{mol})$, BAPTA $(13.9 \mathrm{~kJ} / \mathrm{mol})$, and dibromoBAPTA $(16.9$ $\mathrm{kJ} / \mathrm{mol}$ ) were used. For dimethylBAPTA and dinitroBAPTA, the $\Delta H$ value for BAPTA, $13.9 \mathrm{~kJ} / \mathrm{mol}$, was used because no empirically measured values are available. $\Delta H$ values for these compounds would be expected to be similar to those measured for the related species, and differences on the order of those measured among EGTA, BAPTA, and dibromoBAPTA should have little effect on the final calculated $K_{d}$.

\begin{tabular}{lll}
\hline Table 1. Equilibrium $\mathrm{Ca}^{2+} \boldsymbol{K}_{d} \mathbf{s}$ of chelators & \\
& $\begin{array}{l}K_{d} \text { (standard } \\
\text { conditions*) }\end{array}$ & $\begin{array}{l}K_{d} \text { (cytoplasmic } \\
\text { conditions } \dagger \text { ) }\end{array}$ \\
Chelator & $4.0 \times 10^{-8} \mathbf{M}$ & $1.8 \times 10^{-7} \mathbf{M}$ \\
\hline DimethylBAPTA & $1.0 \times 10^{-7} \mathbf{M}$ & $5.0 \times 10^{-7} \mathbf{M}$ \\
BAPTA & $1.6 \times 10^{-6} \mathbf{M}$ & $4.9 \times 10^{-6} \mathbf{M}$ \\
DibromoBAPTA & $7.0 \times 10^{-3} \mathbf{M}$ & $3.1 \times 10^{-2} \mathbf{M}$ \\
DinitroBAPTA & $9.5 \times 10^{-8} \mathbf{M}$ & $2.2 \times 10^{-7} \mathbf{M}$ \\
EGTA (pH, 7.3) & $3.8 \times 10^{-7} \mathbf{M}$ & $8.6 \times 10^{-7} \mathbf{M}$ \\
EGTA (pH, 7.0) &
\end{tabular}

The calcium of $K_{d}$ of EGTA was calculated for $\mathrm{pH} 7.0$ and $\mathrm{pH} 7.3$, the limits reported for squid cytoplasm (Bicher and Ohki, 1972; Boron and DeWeer, 1976; Adams and Thomas, 1989). No corrections were made for reduction of apparent calcium $K_{d}$ by magnesium, because the affinity of both EGTA (Zucker and Steinhardt 1978; Thomas, 1982) and most of the BAPTA-family buffers (Tsien, 1980) is so much greater for calcium than for magnesium that the presence of scvcral mM free magnesium should have a negligible effect on calcium binding. It is possible that free intracellular magnesium is high enough to bind sufficient dimethylBAPTA to reduce the apparent calcium affinity of this compound (Pethig et al., 1989); however, its calcium affinity should still be greater than that of BAPTA.

* Standard $K_{d} s$ for BAPTA, dimethylBAPTA, and dibromoBAPTA are from Tsien (1980). Standard $K_{d}$ for dinitroBAPTA is from Pethig et al. (1989).

$\dagger$ Cytoplasmic $K_{d} \mathrm{~s}$ were calculated as described in the text.

The buffer $K_{d} s$ under standard conditions and those calculated for $14^{\circ} \mathrm{C}$ at estimated squid cytoplasmic ionic strength are shown in Table 1.

\section{Results}

\section{Differential effects of EGTA and BAPTA on synaptic transmission}

EGTA, injected into the presynaptic terminal at estimated concentrations up to $80 \mathrm{~mm}$, had little or no effect on transmitter release in 10 experiments (Fig. 4). In some experiments, the first

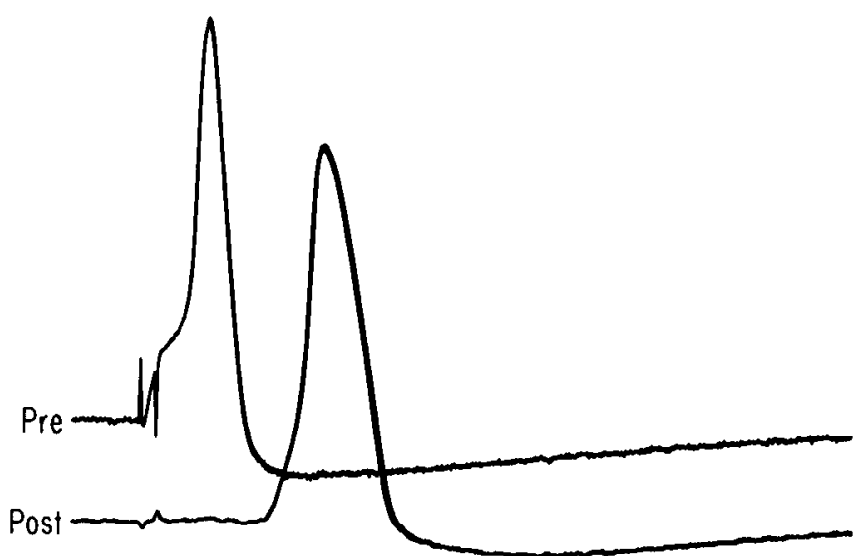

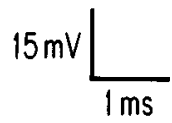

Figure 4. Lack of effect of iontophoresing EGTA into the presynaptic nerve terminal. Five traces of pre- (Pre) and postsynaptic (Post) responses are shown. All five responses consisted of suprathreshold PSPs that triggered action potentials. The first, third, fifth, seventh, and ninth PSPs following initiation of EGTA iontophoresis were completely superimposable. In this unusually small terminal, the presynaptic concentration of EGTA produced by the standard injection protocol was estimated to be $81 \mathrm{~mm}$. 


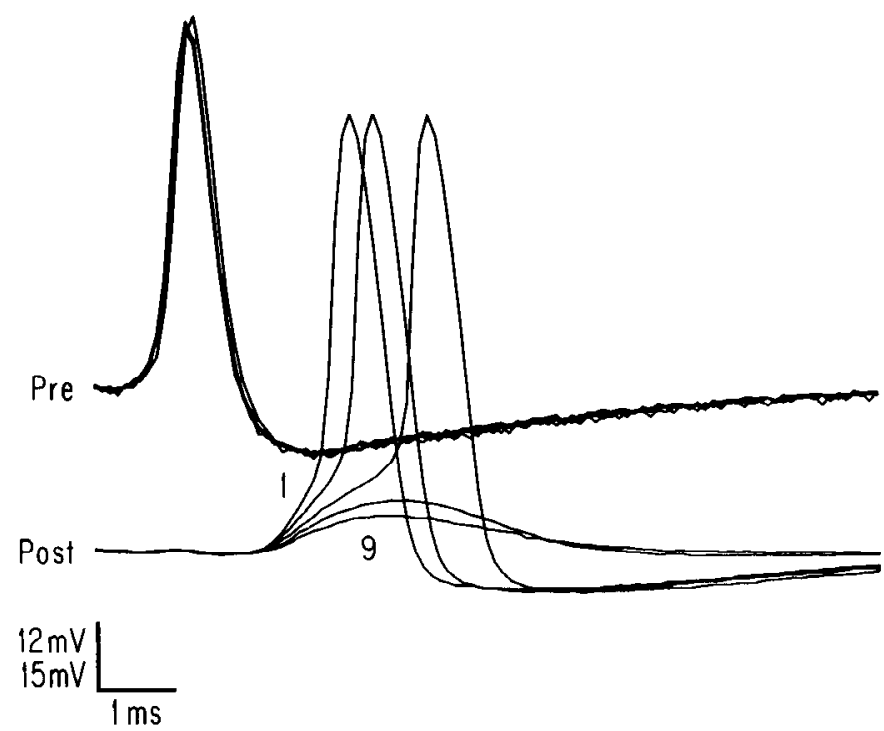

Figure 5. Reduction of the PSP resulting from BAPTA iontophoresis. First, third, fifth, seventh, and ninth responses following initiation of BAPTA iontophoresis showed a progressive reduction in PSP amplitude and $d V / d t$ with continuing iontophoresis of buffer. In this experiment, a slight decrease in presynaptic action potential latency and amplitude (which would lead to an accompanying decrease in PSP latency) was apparent after the first trace; however, the presynaptic spike remained stable thereafter, while the PSP continued to decrease.

stimulus following initiation of iontophoresis resulted in a larger and more rapid presynaptic spike. This was accompanied by a small and transient increase in transmitter output. Presumably, the potentiation of the presynaptic spike and concomitant increase in transmitter output (Miledi and Slater, 1966; Charlton and Bittner, 1978b; Llinas et al., 1982) are caused by membrane potential hyperpolarization leading to a reduction of sodium channel inactivation (Hodgkin and Huxley, 1952). In some experiments, evoked release remained elevated during the entire loading period; in others, it returned to or was slightly below preinjection levels. However, little sustained effect of EGTA injection was seen.

Injection of BAPTA, which has a $K_{d}$ similar to that of EGTA at $\mathrm{pH} 7.2$, into the presynaptic nerve terminal caused a marked reduction of neurotransmitter release in 24 out of 25 experiments. In three of these experiments, an ineffective EGTA injection was followed by an effective BAPTA injection into the same nerve terminal. A reduction in postsynaptic response was apparent within two or three $30-\mathrm{sec}$ iontophoretic sessions at $100 \mathrm{nA}$ (Fig. 5). Transmitter release continued to decrease as more buffer was injected.

A reduction in the amplitude or duration of the presynaptic action potential will reduce neurotransmitter release (Katz and Miledi, 1967; Llinas et al., 1982). However, BAPTA iontophoresis did not attenuate the presynaptic action potential; therefore, this was not a factor in the present results. There was sometimes a transient increase in presynaptic spike amplitude and transmitter release following initiation of BAPTA iontophoresis, as with EGTA. Following this initial increase, transmitter release to subsequent stimuli declined with continued BAPTA iontophoresis despite a maintained increase in the presynaptic action potential. In other experiments, release began to decline immediately after initiation of iontophoresis.

After cessation of iontophoresis, transmitter release often continued to decline for several minutes. This probably was due to a redistribution of buffer within the nerve terminal, leading to an increase in the number of release sites at which calcium was chelated (see Fig. 3), though other explanations (e.g., residual effects of sustained hyperpolarization) are certainly possible. This decline was transient and was followed by a slow recovery toward preinjection levels (Fig. 6). This cycle of buffer injection, release depression, and release recovery could be repeated several times in a given synapse (Fig. 6). The estimated buffer concentration following cessation of iontophoresis at maximum PSP reduction was several millimolar (see below).

A pharmacological agent could inhibit transmitter release, with little or no effect on the presynaptic action potential, by reducing calcium entry into the nerve terminal. In order to

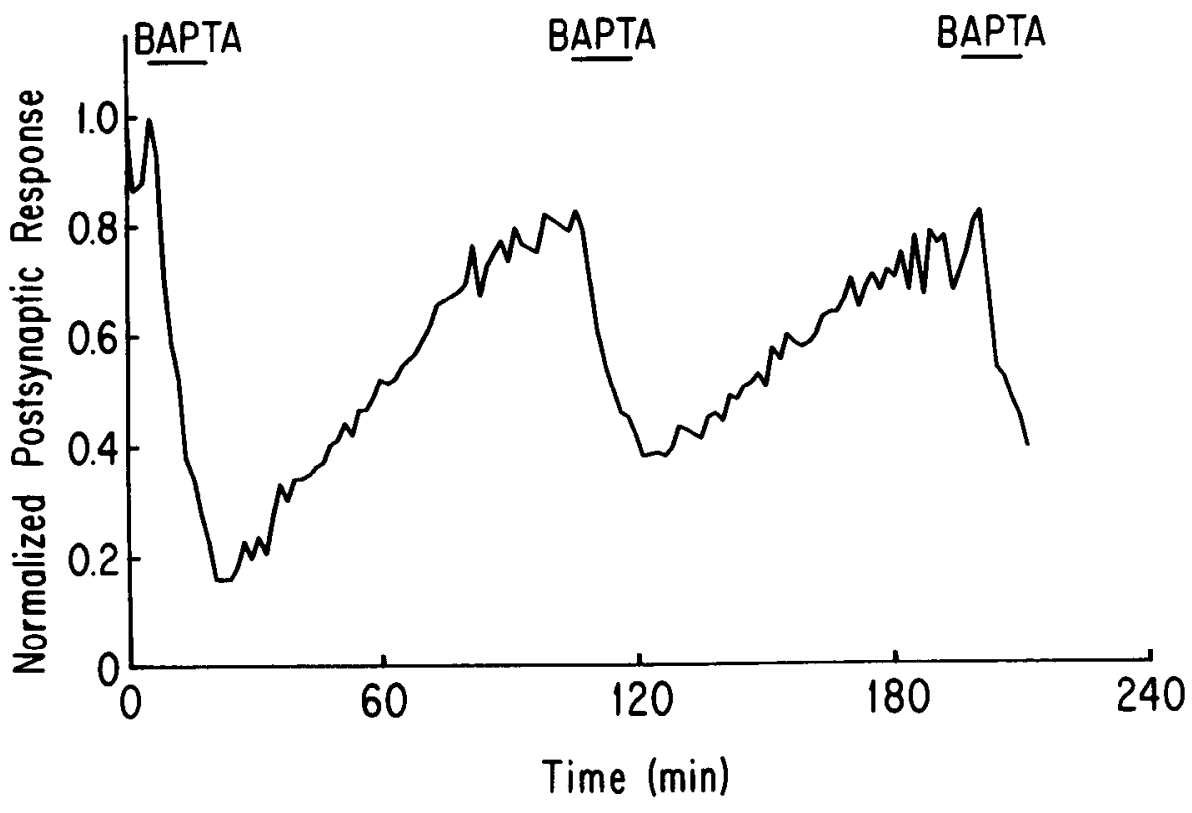

Figure 6. The time course and reversibility of BAPTA-induced attenuation of synaptic transmission. Postsynaptic responses were quantified by measuring the rate of rise of the PSP and were normalized by dividing by the maximum rate of rise measured. BAPTA iontophoresis took place at the times indicated by the bars. 
Figure 7. A, Calcium spikes (Pre) and PSPs (Post) elicited before and after BAPTA iontophoresis. BAPTA iontophorcsis caused a profound reduction in the amount of transmitter released even though the calcium spike was actually larger and longer than the control calcium spike. The increase in calcium spike amplitude and duration occurred because the blockade of $K$ channels by DAP had not entirely reached equilibrium at the time BAPTA was injected. $B$, Reduction of calcium spikes and PSPs by cadmium $(C d)$. Data were recorded during equilibration of $100 \mu \mathrm{M}$ cadmium before its full effects occurred. A much more moderate reduction in transmitter relcase than that produced by BAPTA was accompanied by a clear decrease in the calcium spike. The traces in $B$ are from the same experiment as the traces shown in $A$ and were collected during recovery from BAPTA iontophoresis. In both $A$ and $B$, the traces begin at a time following the presynaptic stimulating pulses.
A

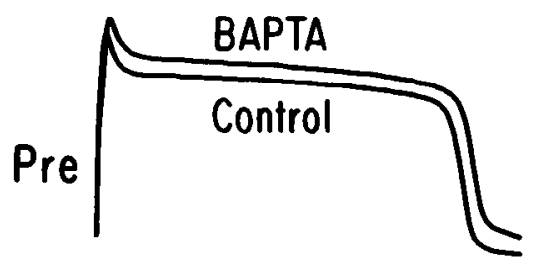

B
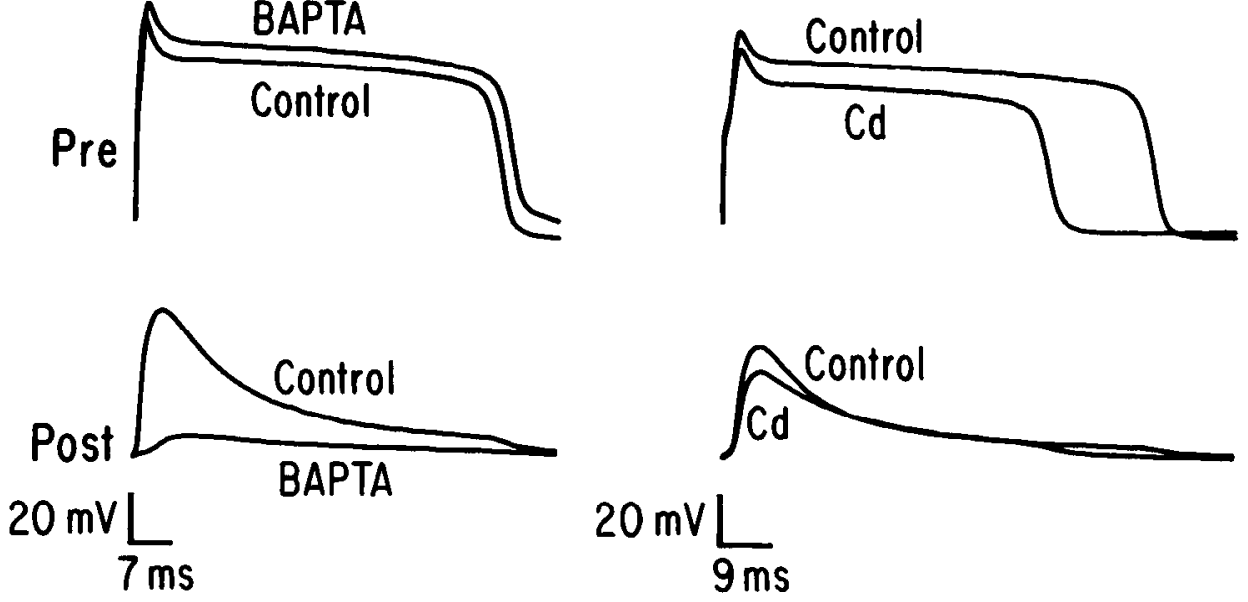

investigate this possible mechanism of BAPTA action, calcium spikes were elicited in the presence of tetrodotoxin (TTX; $1 \mu \mathrm{M}$ ) and 3,4-diaminopyridine (DAP; $2 \mathrm{~mm}$ ) to block sodium and potassium conductances, respectively (Katz and Miledi, 1967; Llinas et al., 1976). Under these conditions, BAPTA caused a pronounced attenuation of transmitter release throughout the entire calcium spike, but no reduction in the amplitude or duration of the calcium spike was apparent (Fig. $7 A$ ). In order to determine whether it was possible that a reduction in calcium conductance sufficient to reduce transmitter release might not be apparent as a reduction in the calcium spike, the calcium conductance was partially blocked with cadmium, a known blocker of presynaptic calcium channels in this preparation (Llinas et al., 1981; Charlton et al., 1982; Augustine and Eckert, 1984), and the resulting attenuation of transmitter release was compared to that produced by BAPTA. Because the object of this experiment was to observe the effect on the calcium spike of a concentration of cadmium that produced only a modest reduction in transmitter release (i.e., only partial block of calcium current), data were recorded during equilibration of 100 $\mu \mathrm{M}$ cadmium before its full effects occurred. Cadmium markedly reduced both the amplitude and duration of the presynaptic calcium spike while producing a much more moderate reduction in transmitter release than BAPTA (Fig. $7 B$ ). While it is possible that BAPTA could cause a decrease in inward calcium current that was exactly balanced by a simultaneous decrease in an outward current with no net reduction of the spike, it seems more likely that the lack of BAPTA effect on the presynaptic calcium spike indicates that BAPTA has little, if any, effect on the calcium current. We therefore provisionally conclude that BAPTA attenuates transmitter release at a step subsequent to calcium entry into the nerve terminal.

\section{Effect of BAPTA derivatives with different calcium affinities}

To investigate further those buffer attributes necessary to attenuate release, the effects of several BAPTA derivatives with different calcium affinities were determined. The effects of dimethylBAPTA $\left(0.18 \mu \mathrm{M}\right.$ estimated $K_{d}$ in axoplasm), dibromo-
BAPTA ( $4.9 \mu \mathrm{M}$ estimated $K_{d}$ ), and dinitroBAPTA (31 mM estimated $K_{d}$ ) were compared. These compounds were selected because their affinities cover a wide range on both sides of BAPTA's $K_{d}$, which is estimated as $0.5 \mu \mathrm{M}$.

All four compounds were capable of reducing transmitter release when injected into the presynaptic terminal (Fig. 8). The mean reduction in transmission produced by dimethylBAPTA (average estimated concentration, $3.9 \mathrm{mM}$ ) was $79 \pm 4.9 \%(n$ $=5$; mean $\pm \mathrm{SEM}$ ), while the mean reduction produced by dibromoBAPTA (average estimated concentration, $3.8 \mathrm{~mm}$ ) was $95 \pm 1.8 \%(n=4)$. BAPTA itself (estimated concentration, 4 $\mathrm{mM})$ reduced transmitter release by $76 \pm 7.2 \%(n=6)$. Dinitro-

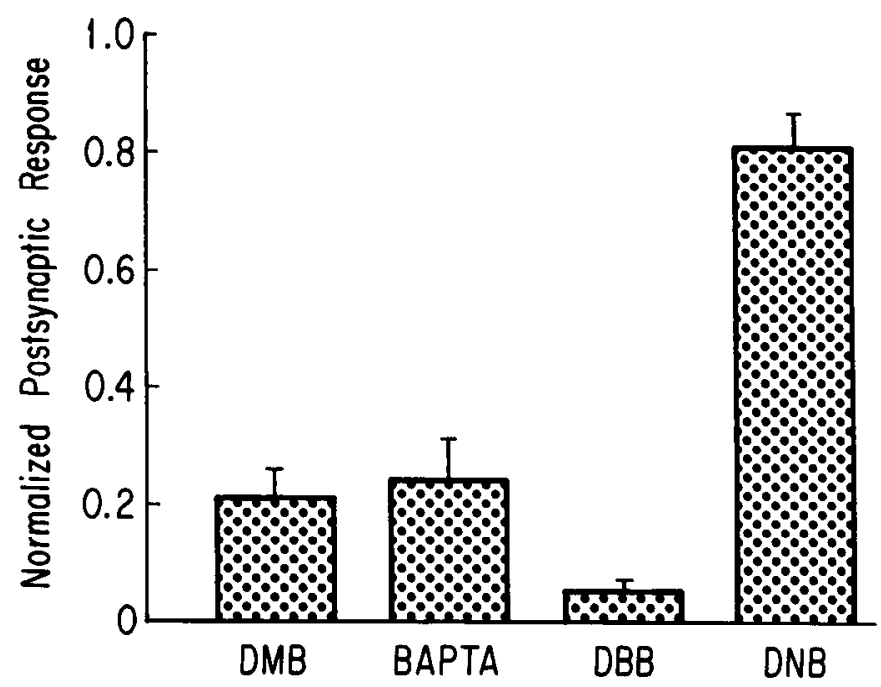

Figure 8. Relative ability of several BAPTA derivatives to reduce transmitter release. The concentration of each buffer in the presynaptic terminal was about $4 \mathrm{~mm}$. Values for postsynaptic responses are means (4-7 replicates for each buffer), normalized relative to measurements made before buffer injection, and error bars indicate SEM. 


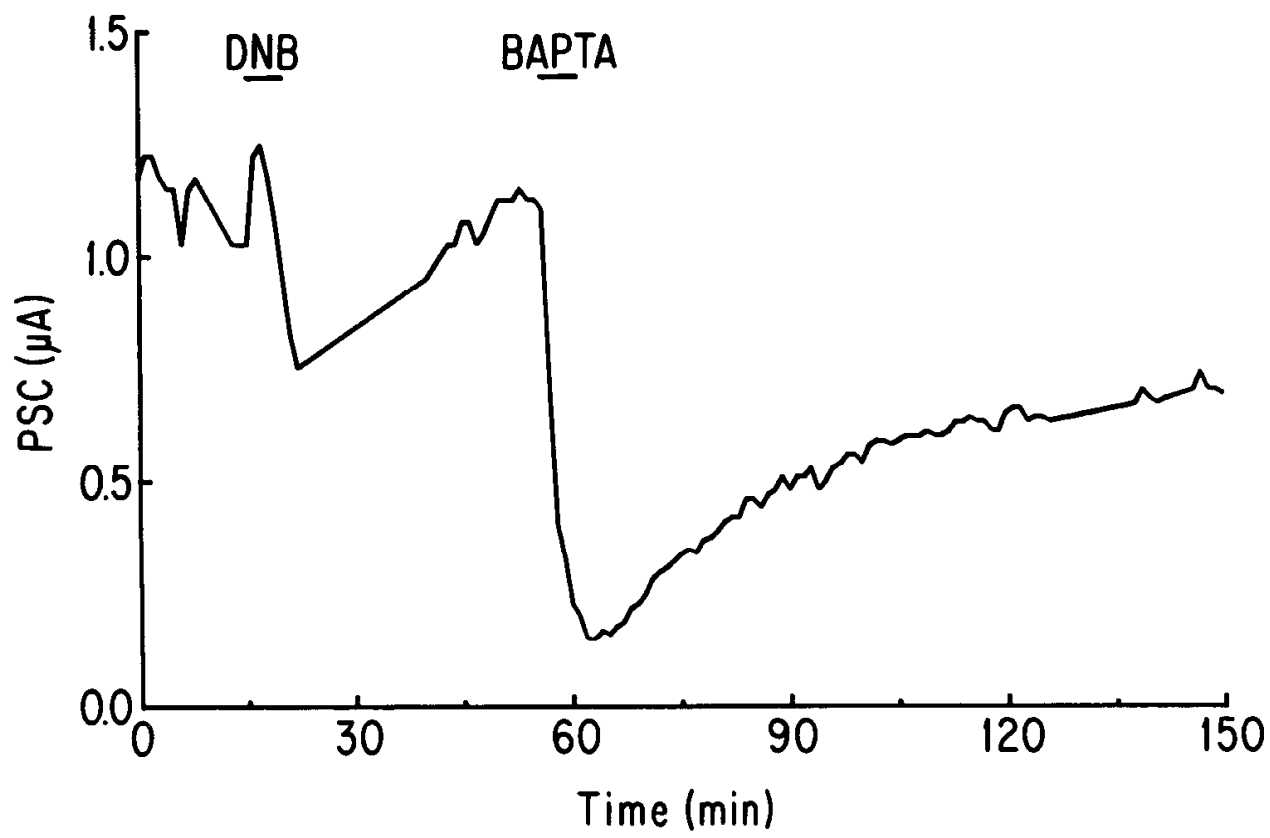

Figure 9. Comparison of the rcduction in transmitter release produced by dinitroBAPTA $(D N B)$ and BAPTA in jection into the same nerve terminal. Of seven experiments in which the effects of dinitroBAPTA were examined, the greatest attenuation of transmitter release was observed in the experiment shown here. Both dinitroBAPTA and BAPTA were injected to a concentration of about $3.9 \mathrm{~mm}$ in this experiment; dinitroBAPTA concentration was estimated to have declined to about 480 $\mu \mathrm{M}$ at the time of BAPTA iontophoresis.
BAPTA was far less effective in reducing transmitter release. In two experiments, no apparent reduction of release was seen following dinitroBAPTA iontophoresis (estimated maximum concentration, 6.7 and $7.6 \mathrm{~mm}$ ). In five additional experiments, where dinitroBAPTA iontophoresis was followed by BAPTA iontophoresis into the same nerve terminal, dinitroBAPTA caused a reduction of $27 \pm 7.5 \%$ in transmitter release (average estimated concentration, $2.8 \mathrm{~mm}$ ), while similar concentrations of BAPTA ( $2.6 \mathrm{~mm})$ produced an $80 \pm 5.8 \%$ reduction in transmitter release (Fig. 9).

The rate of recovery of transmitter release following similar injections of the various buffers was different. Recovery of transmission after injection of dimethylBAPTA was much faster than recovery following BAPTA injection, while recovery from dibromoBAPTA was somewhat slower. If recovery results from diffusion of buffer out of the nerve terminal and if all of these compounds have similar diffusion coefficients, this difference in recovery rate suggests that slight differences in buffer efficacy are accentuated at low buffer concentrations. To examine this possibility, buffer concentration was estimated during recovery from iontophoresis, and the percent reduction of the PSP at various concentrations of the different buffers was compared. We did not analyze buffer effects during the loading period because the inhomogeneous distribution of buffer in the terminal at this time complicates the analysis.

After cessation of iontophoresis, injected buffer will diffuse out of the terminal and into the preterminal axon (see Fig. $2 A$ ). A simple approximation was used to estimate presynaptic buffer concentration after injection. In this approximation, the axon was treated as a semi-infinite cylinder (of cross-sectional area 4) of twice the diameter measured at the base of the nerve terminal with entire estimated terminal buffer content diffusing from a plane at one end of the cylinder starting at time 0 . Equation 2 was normalized for actual nerve terminal diameter and solved for $x=0$ (see Atkins, 1978, for this solution of Fick's second law).

Because buffer concentration was being determined for a small compartment of known dimensions, rather than at a plane at the junction between the nerve terminal and the presynaptic axon, the calculated volume of the nerve terminal was added to the estimated volume of axonal diffusion in order to calculate average concentration in the nerve terminal:

$$
C_{(\text {terminal }, t)}=N_{(0)} /\left(\text { terminal volume }+A(\pi D t)^{1 / 2}\right) .
$$

In this approximation, the component of buffer that diffuses directly from the injection site to the closed tip of the terminal was ignored because this compartment is so small that it should have relatively little impact except at very early times during and immediately after loading. Similarly, buffer deposition was assumed to be instantaneous.

The value of $2 \times 10^{-6} \mathrm{~cm}^{2} \mathrm{sec}^{-1}$ (Neher, 1986), was used for the diffusion coefficient, $D$, for all buffers. This is very similar to $D$ measured for dinitroBAPTA (see Materials and Methods), and it is unlikely that the other buffers, which are closely related chemically, would show marked differences in $D$. Moreover, the onset of action of the three lower $K_{d}$ buffers was rapid, as expected if diffusion of buffer was unimpeded.

In general, differences in calcium buffer efficacy in suppressing transmitter release became much more apparent at buffer concentrations less than $1 \mathrm{~mm}$ (Fig. 10). Even at concentrations greater than $1 \mathrm{mM}$, a clear reduction in buffer efficacy was apparent with the very high- $K_{d}$ buffer dinitroBAPTA (Fig. 8). On average, a $50 \%$ reduction in PSP amplitude was produced by $0.87 \pm 0.17 \mathrm{~mm}$ dimethylBAPTA $(n=5)$ and $0.73 \pm 0.20 \mathrm{~mm}$ BAPTA $(n=5)$. Less than $0.3 \mathrm{~mm}$ dibromoBAPTA was required to produce an equivalent reduction, because transmitter release had not recovered to $50 \%$ of control values at this estimated concentration $(n=4)$. In six out of seven experiments, millimolar concentrations of dinitroBAPTA (average concentration, $4 \mathrm{~mm}$ ) were insufficient to cause a $50 \%$ reduction in transmitter release. Thus, under these conditions, the relative efficacy of these compounds was dibromoBAPTA $>$ BAPTA $\geq$ dimethylBAPTA > dinitroBAPTA. This differs from the efficacy of these compounds in binding calcium ions (Table 1). 


\section{Discussion}

\section{Calcium buffers block transmitter release}

This study demonstrates that several members of the BAPTA family of $\mathrm{Ca}^{2+}$ buffers are capable of reducing evoked neurotransmitter release when injected into the presynaptic terminal of the squid giant synapse, while EGTA has little or no effect. BAPTA derivatives with estimated intracellular calcium $K_{d} \mathrm{~s}$ ranging from 0.18 to $4.9 \mu \mathrm{M}$ were all highly effective at reducing transmitter release at intraterminal concentrations estimated to be in the millimolar range. A BAPTA derivative with an estimated $K_{d}$ of $31 \mathrm{~mm}$ was much less effective. These results confirm and extend the observation of Adams et al. (1985) that presynaptic EGTA injection has little effect on evoked transmitter release at the squid giant synapse. They are also consistent with the report that a cell-permeant derivative of BAPTA reduces transmitter release at the crayfish neuromuscular junction (Charlton and Iwanchyshyn, 1986).

The results cannot be interpreted as secondary to pharmacologic effects on the nerve terminal action potential or calcium conductance. While other nonspecific pharmacological effects cannot be rigorously excluded and have been suggested for dibromoBAPTA in mast cells (Penner and Neher, 1988) and for dinitroBAPTA in fucus eggs (Pethig et al., 1989), it is more likely that the reductions in transmitter release caused by these buffers are consequent to their known ability to bind calcium and to the resulting attenuation of the presynaptic calcium transient that triggers release.

\section{Lack of EGTA effect defines spatiotemporal constraints of calcium receptor binding}

On the basis of calcium affinity alone, the efficacy of EGTA in reducing transmitter release should be similar to that of the BAPTA-family buffers because the $K_{d}$ for EGTA at pH 7.3 falls between that of dimethylBAPTA and BAPTA, while at $\mathrm{pH} 7.0$ it falls between BAPTA and dibromoBAPTA. Several interpretations of this observation are possible. It is possible that EGTA iontophoresis was ineffectual and that the lack of EGTA effect was due to a lack of presynaptic EGTA. This is unlikely because Adams et al. (1985) found that EGTA iontophoresis restored release in metabolically poisoned nerve terminals in which calcium was pathologically elevated. Very recently, Swandulla et al. (1991) have also found that EGTA iontophoresis blocks slow tetanic rises in calcium concentration in the terminal. This suggests that EGTA both gets into the nerve terminal and, once there, has some ability to buffer calcium.

Because calcium/EGTA association is accompanied by the dissociation of two protons, it is possible that lack of EGTA effect could result from an increase in transmitter release secondary to local cytoplasmic acidification, which counteracts the effect of EGTA on the calcium transient. However, Adams and Thomas (1989) and J. Dietmer, K. Zipser, and G. J. Augustine (unpublished observations) have found little effect of reductions in presynaptic $\mathrm{pH}$ on transmitter release at the squid giant synapse. It thus seems unlikely that a compensatory increase in free $\mathrm{H}^{+}$ions could be responsible for the lack of effect of EGTA on transmitter release.

The most likely explanation is that the kinetics of EGTA/ calcium binding are slow relative to the initiation of transmitter release, so that EGTA may be unable to reduce calcium concentration rapidly enough to reduce transmitter release in this synapse. A similar explanation has been proposed to account

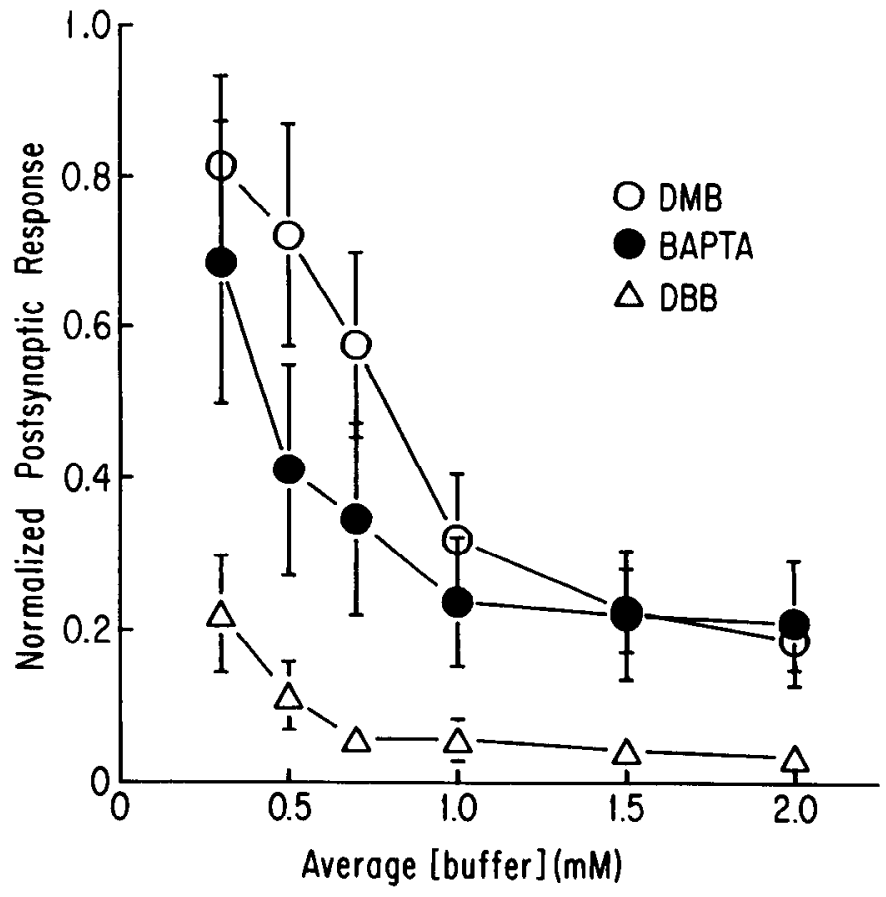

Figure 10. The relative efficacy of various BAPTA derivatives in reducing postsynaptic responses at different average buffer concentrations, estimated during recovery from iontophoresis. PSP rate of rise was measured at $1-\mathrm{min}$ intervals during the first $10 \mathrm{~min}$ after cessation of iontophoresis, and at 10-min intervals thereafter for 40-60 $\mathrm{min}$, and plotted against estimated average concentration. In those experiments in which release continued to decline after cessation of iontophoresis, time 0 for recovery was taken as the time of maximum PSP reduction. Lines were drawn by eye through the points thus obtained, and values for PSP reduction in individual experiments were determined for 0.3 , $0.5,0.7,1.0,1.5$, and $2.0 \mathrm{~mm}$ estimated buffer concentration. Normalized values for PSP reduction from different experiments were then averaged. In several experiments, values of PSP reduction were extrapolated from values obtained at higher concentrations: in two experiments, recovery from dimethylBAPTA $(D M B)$ was complete at concentrations greater than $300 \mu \mathrm{M}$, and recovery was not followed further; $100 \%$ recovery was then extrapolated to the lower concentrations. A few other experiments were terminated when estimated buffer concentration was greater than $300 \mu \mathrm{M}$; in two of these experiments, estimated concentration was greater than $500 \mu \mathrm{M}$. Straight lines were drawn through the recovery points obtained (over at least 40 min recovery time and starting after the end of the flat portion of the recovery curve), and values for the lower concentration were extrapolated from these lines. Most of the approximations used in calculating estimated concentration (see Materials and Methods) will be reasonably accurate at times greater than 10 min after ceasing iontophoresis (corresponding to average concentrations less than $1 \mathrm{~mm}$ on this graph). Concentrations obtained at earlier times will be distorted by several factors that will have minimal impact at longer times: inhomogeneity of buffer distribution within the nerve terminal, slight errors in determining the 0 time of recovery, loss of buffer during the latter part of iontophoretic loading, and the assumption that buffer deposition in the nerve terminal was instantaneous. Because the average concentration at the end of loading of BAPTA, dimethylBAPTA, and dibromoBAPTA $(D B B)$ was aboul the same, it is still reasonable to compare the effects of the three different buffers during this time frame, even though the actual values for concentration are somewhat imprecise. Points represent mean $\pm \operatorname{SEM}(n=4$ for each buffer).

for differences in the ability of EGTA and BAPTA to attenuate calcium-activated $\mathrm{K}$ currents in chromaffin cells (Marty and Neher, 1985; Neher, 1986). The possible role of calcium-binding kinetics in the differential actions of EGTA and BAPTA can be seen by considering the relative speed of equilibration of the 
two buffers at a conservative estimate (e.g., $10 \mathrm{~mm}$ ) of the presynaptic EGTA concentrations attained. The specific form of the relaxation equations used and therefore of the time constants derived are only appropriate under conditions where the association of buffer and calcium is the only factor perturbing their free concentrations. This is obviously not the case for neurotransmitter release because the calcium concentration in the vicinity of release sites is rapidly changing (e.g., Simon and Llinas, 1985; Zucker and Fogelson, 1986). However, despite this limitation, the relative impact of differences in buffer kinetics upon the presynaptic calcium transient should be preserved.

The reaction

$$
[\mathrm{Ca}]+[\text { Buffer }] \underset{k_{\text {ofr }}}{\stackrel{k_{\text {on }}}{\rightleftharpoons}}[\mathrm{CaBuffer}]
$$

will approach equilibrium at a rate that depends on the concentration of the reactants and the forward and reverse rate constants ( $k_{\text {on }}$ and $k_{\text {off }}$ ) of the reaction. Under conditions where one of the reactants is present in great excess over the other, so that its concentration will remain quasi-constant throughout the reaction (as would surely be the case for a buffer concentration of $10 \mathrm{~mm}$ or greater), the time constant, $\tau$, of buffer/calcium equilibration will be given by

$$
1 / \tau=k_{\text {on }}\left[\text { Buffer }_{\text {total }}\right]+k_{\text {off }} \text {, }
$$

where $\tau$ defines the relaxation time of the reaction (see Bernasconi, 1976). The forward and backward rate constants for the EGTA/calcium reaction are $1.5 \times 10^{6} \mathrm{M}^{-1} \mathrm{sec}^{-1}$ and $0.3 \mathrm{sec}{ }^{1}$, respectively (at $\mathrm{pH} 7,25^{\circ} \mathrm{C}, 0.1 \mathrm{M}$ ionic strength; Smith et al., 1984). For a concentration of $10 \mathrm{~mm}$ EGTA, Equation 10 gives a value for $\tau$ of $67 \mu \mathrm{sec}$. Therefore, the reaction will be close to equilibrium in about $200 \mu \mathrm{sec}$, under these conditions of temperature and ionic strength. In contrast, if BAPTA is assumed to have binding kinetics similar to those of fura-2 $\left(k_{\text {on }}\right.$ of 6.02 $\times 10^{8} \mathrm{M}^{-1} \mathrm{sec}^{-1}, k_{\text {off }}$ of $96.7 \mathrm{sec}^{-1}$ at $20^{\circ} \mathrm{C}, \mathrm{pH} 7.4-8.4,0.14 \mathrm{M}$ ionic strength; Kao and Tsien, 1988), $\tau$ for $10 \mathrm{~mm}$ BAPTA is about $0.17 \mu \mathrm{sec}$, so that this reaction will be close to equilibrium in about $0.5 \mu \mathrm{sec}$.

Under squid cytoplasmic conditions, both reactions will be considerably slowed because of temperature, ionic strength, and, possibly, viscosity. Baylor and Hollingworth (1988) have estimated that, in frog skeletal muscle, the $k_{\text {on }}$ for the reaction between calcium and fura- 2 is slowed 6-60-fold relative to the value obtained under standard conditions. Because much of this reduction in calcium binding rate is apparently secondary to fura-2 binding to myoplasmic constituents (Baylor and Hollingworth, 1988) and the $D$ measured for dinitroBAPTA suggests little, if any, cytoplasmic binding of BAPTA compounds in squid axoplasm (see Materials and Methods), a sixfold reduction in $k_{\text {on }}$ is probably an upper limit to the slowing of calcium binding to these compounds under our conditions. In this case, the EGTA/calcium reaction will be close to equilibrium in $1.2 \mathrm{msec}$, and the BAPTA/calcium reaction will be close to equilibrium in $3 \mu \mathrm{sec}$. Because the delay between influx of calcium and initiation of transmitter release is about $200 \mu \mathrm{sec}$ (Llinas et al., 1981; Augustine et al., 1985), it is clear that over this time interval BAPTA will bind more calcium than will EGTA, and this presumably accounts for the observed differential effects of these buffers on release.
The above estimate of $1.2 \mathrm{msec}$ for equilibration of calcium binding to EGTA suggests that EGTA should still reduce free calcium (and hence transmitter release) to some extent within the $200-\mu \mathrm{sec}$ minimal synaptic delay. As Equation 10 indicates, the equilibration time will be even shorter at buffer concentrations greater than $10 \mathrm{~mm}$. The lack of EGTA effect indicates that a considerable portion of the minimal synaptic delay occurs after calcium diffuses and binds to the receptor molecule(s) responsible for triggering release. For example, if the diffusion and binding steps occurred within 10-20 $\mu \mathrm{sec}$, very little EGTA effect would be apparent, but the BAPTA reaction would still be close to or at equilibrium. Another implication of these kinetic considerations is that the calcium receptor is located very close to the calcium channels, because calcium will diffuse only about $63 \mathrm{~mm}$ in $10 \mu \mathrm{sec}$, if it has a diffusion coefficient of $2 \times$ $10^{-6} \mathrm{~cm}^{2} \mathrm{sec}^{-1}$ (Augustine et al., 1987). This is consistent with recent indications that calcium channels are closely associated with the sites of release (Augustine et al., 1989).

\section{Differential effects of chelator affinity require a large calcium transient}

The discussion in the previous section suggests that an equilibrium analysis may be appropriate when considering the actions of BAPTA on transmitter release. This may also be true for the BAPTA derivatives we have used: available estimates of calcium association rates for members of the BAPTA family differ by a factor of less than three over a 50 -fold range of $K_{d}$ (Smith et al., 1983; Quast et al., 1984; Jackson et al., 1987; Kao and Tsien, 1988) and are hundreds of times greater than the calcium association rate of EGTA (Smith et al., 1984).

In the case of equilibrium calcium binding, the order of buffer efficacies will depend on the buffer $K_{d}$, the concentration of buffer, and the intracellular concentration of free calcium, $[\mathrm{Ca}]_{i}$, during the transient (for the equations describing this relationship, see Zucker and Steinhardt, 1978; Nachsen and Drapeau, 1982). For a given calcium load, the order of buffer efficacy should roughly parallel the affinity for calcium, unless $[\mathrm{Ca}]_{i}$ exceeds the intracellular buffer concentration, in which case differences among the three high-affinity buffers (dimethylBAPTA, BAPTA, dibromoBAPTA) should be negligible. However, the order observed in blocking release did not match either of these predictions.

One possible explanation for this discrepancy is that the effective concentration of buffer available to chelate calcium entering during the transient is reduced by binding to free calcium in the resting nerve terminal $\left([\mathrm{Ca}]_{\text {rest }}\right)$ prior to nerve stimulation. With a $[\mathrm{Ca}]_{\text {rest }}$ of 10-100 nM (Baker and DiPolo, 1984), the reduction in free buffer concentration for a buffer load of 300 $\mu \mathbf{M}$ to several $\mathbf{m M}$ is negligible if the chelation of $[\mathrm{Ca}]_{\text {rest }}$ is uncompensated and $[\mathrm{Ca}]_{\text {rest }}$ is allowed to drop to levels on the order of $10^{-9} \mathrm{M}$. However, studies in other systems (see references in Tsien et al., 1984) have suggested that cellular homeostatic mechanisms can maintain $[\mathrm{Ca}]_{\text {rest }}$ at a "set point" near normal levels despite the presence of several mм of alien calcium buffer. For example, Baker and Umbach (1987) have found that injecting EGTA and BAPTA into squid axoplasm (at concentrations below $5 \mathrm{mM}$ ) produces only a transient drop in $[\mathrm{Ca}]_{\text {rest }}$. This suggests that exogenous buffers may become partially saturated by resting calcium, so that a fraction of the buffer will be unavailable to chelate the calcium entering during the transient. In this case, the amount of free buffer will be inversely related to the calcium affinity. 
If $[\mathrm{Ca}]_{\text {rest }}$ is assumed to be set at $100 \mathrm{~nm}$ (Baker and DiPolo, 1984), calculations indicate that the predicted order of buffer efficacy resembles that observed only if $[\mathrm{Ca}]_{i}$ is several hundred $\mu \mathrm{M}$ or greater (E. M. Adler, G. J. Augustine, and M. P. Charlton, unpublished calculations). If an additional, native calcium buffer (Baker and Schlaepfer, 1978; Krinks et al., 1988) is included, this value rises into the $\mathrm{mm}$ range, depending on the calcium affinity and concentration of the endogenous buffer. Thus, the effects of calcium buffers suggest that transmitter release may be activated by $[\mathrm{Ca}]_{i}$ of hundreds of $\mu \mathrm{M}$ or more.

Estimates of $[\mathrm{Ca}]_{i}$ in the transmitter releasing region of frog hair cells also suggest that calcium levels may reach hundreds of $\mu \mathrm{M}$ or more during depolarization (Roberts et al., 1990), and it appears that calcium concentration at secretory sites of chromaffin cells reaches $10-100 \mu \mathrm{M}$ during depolarization (G. J. Augustine and E. Neher, unpublished observations). This high concentration of calcium could be found very close to a single open calcium channel or within the area of overlap of calcium coming from a cluster of open channels (Fogelson and Zucker, 1985; Simon and Llinas, 1985; Smith and Augustine, 1988). Production of high $[\mathrm{Ca}]_{i}$ levels at release sites suggests that the calcium receptor that initiates release does not need to have a high affinity for calcium.

A very similar pattern of BAPTA-family buffer efficacy has been described in inhibiting fucoid egg development (Speksnijder et al., 1989). These results have been interpreted as resulting from buffer suppression of intracellular calcium gradients by shuttling calcium from regions of high concentration to regions of low concentration. This mechanism is less likely to explain buffer attenuation of transmitter release because of the extremely rapid nature of the transmitter release reaction. We instead propose that our results are due to a relatively large calcium transient and partial occupancy of the higher-affinity buffers by calcium ions prior to nerve stimulation.

In summary, the relative effectiveness of a calcium chelator in a given system will depend on a number of factors, including the concentration of calcium and chelator, the diffusion distance between calcium sources and receptors, and the kinctics of rcactions involving both exogenous and endogenous molecules (see also Neher, 1986). An implication of our results is that EGTA and other slowly binding calcium buffers can give false negative results when used as a diagnostic tool to examine a rapid calcium-mediated process.

\section{Conclusion}

These results provide additional evidence in favor of the calcium hypothesis of neurotransmitter release by demonstrating that calcium must not only enter the nerve terminal in order to trigger neurotransmitter release, but must also diffuse to the appropriate site to do so. Consideration of the relative efficacy of the various chelators in attenuating release suggests that the presynaptic calcium transient that triggers release may bc large, perhaps hundreds of $\mu \mathrm{M}$ or more. The calcium receptor that triggers release binds calcium rapidly (on the order of microseconds) appears to be close to calcium channels and could have a low affinity for calcium. These inferences place constraints on the location and functional characteristics of the calcium-binding molecules that initiate transmitter release.

\section{References}

Adams DJ, Thomas MV (1989) Effects of sodium azide and cytosolic $\mathrm{pH}$ on evoked transmitter release at the isolated squid giant synapse. J Physiol 410:82P.
Adams DJ, Takeda K, Umbach JA (1985) Inhibitors of calcium buffering depress evoked transmitter release at the squid giant synapse. J Physiol (Lond) 369:145-159.

Adler EM, Augustine GJ, Duffy SN, Charlton MP (1988a) Modulation of neurotransmitter release by intracellular calcium chelators at the squid giant synapse. Soc Neurosci Abstr 14:69.

Adler EM, Duffy SN, Augustine GJ, Charlton MP (1988b) Effects of intracellular alien calcium chelators on transmitter release at the squid giant synapse. Biol Bull 175:312-313.

Atkins PW (1978) Physical chemistry. San Francisco: Freeman.

Augustine GJ, Eckert R (1984) Divalent cations differentially support transmitter release at the squid synapse. J Physiol (Lond) 346:257271.

Augustine GJ, Charlton MP, Smith SJ (1985) Calcium entry and transmitter release at voltage-clamped nerve terminals of squid. J Physiol (Lond) 369:163-181.

Augustine GJ, Charlton MP, Smith SJ (1987) Calcium action in synaptic transmitter release. Annu Rev Neurosci 10:633-693.

Augustine GJ, Buchanan J, Charlton MP, Osses LR, Smith SJ (1989) Fingering the trigger for neurotransmitter secretion: studies on the calcium channels of squid giant presynaptic terminals. In: Sccretion and its control (Oxford GS, Armstrong CM, eds), pp 203-223. New York: Rockefeller UP.

Baker PF, DiPolo R (1984) Axonal calcium and magnesium homeostasis. Curr Top Membr Transp 22:195-247.

Baker PF, Shlaepfer WW (1978) Uptake and binding of calcium by axoplasm isolated from giant axons of Loligo and Myxicola. J Physiol (Lond) 276:103-125.

Baker PF, Umbach JA (1987) Calcium buffering in axons and axoplasm of Loligo. J Physiol (Lond) 383:369-394.

Baylor SM, Hollingworth S (1988) Fura-2 calcium transients in frog skeletal muscle fibres. J Physiol (Lond) 403:151-192.

Bernasconi CF (1976) Relaxation kinetics. New York: Academic.

Bicher HI, Ohki S (1972) Intracellular $\mathrm{pH}$ electrode experiments on the giant squid axon. Biochim Biophys Acta 255:900-904.

Boron WF, DeWeer P (1976) Intracellular pH transients in squid giant axons caused by $\mathrm{CO}_{2}, \mathrm{NH}_{3}$, and metabolic inhibitors. J Gen Physiol 67:91-112.

Charlton MP, Bittner GD (1978a) Facilitation of transmitter release at squid synapses. J Gen Physiol 72:471-486.

Charlton MP, Bittner GD (1978b) Presynaptic potentials and facilitation of transmitter release in the squid giant synapse. J Gen Physiol $72: 487-511$

Charlton MP, Iwanchyshyn G (1986) Exogenous calcium buffer reduces synaptic transmitter release and facilitation. Soc Neurosci Abstr 12:817.

Charlton MP, Smith SJ, Zucker RS (1982) Role of presynaptic calcium ions and channels in synaptic facilitation and depression at the squid giant synapse. J Physiol (Lond) 323:173-193.

Davies CW (1962) Ion association. London: Butterworths.

Fogelson AL, Zucker RS (1985) Presynaptic calcium diffusion from various arrays of single channels. Implications for transmitter release and synaptic facilitation. Biophys J 48:1003-1017.

Gainer H, Gallant PE, Gould R, Pant HC (1984) Biochemistry and metabolism of the squid giant axon. Curr Top Membr Transp 22: $57-90$.

Harrison SM, Bers DM (1987) The effect of temperature and ionic strength on the apparent $\mathrm{Ca}$-affinity of EGTA and the analogous $\mathrm{Ca}$ chelators BAPTA and dibromo-BAPTA. Biochim Biophys Acta 925: 133-143.

Hodgkin AL, Huxley AF (1952) A quantitative description of membrane current and its application to conduction and excitation in nerve. J Physiol (Lond) 117:500-544.

Jackson AP, Timmerman MP, Bagshaw CR, Ashley CC (1987) The kinetics of calcium binding to fura-2 and indo-1. FEBS Lett 216:3539.

Kao JPY, Tsien RY (1988) $\mathrm{Ca}^{2+}$ binding kinetics of fura-2 and azo-1 from temperature-jump relaxation measurements. Biophys J 53:635639.

Katz B (1969) The release of neural transmitter substances. Liverpool: Liverpool UP.

Katz B, Miledi R (1967) A study of synaptic transmission in the absence of nerve impulses. J Physiol (Lond) 192:407-436.

Krinks MH, Klee CB, Pant HC, Gainer H (1988) Identification and quantification of calcium-binding proteins in squid axoplasm. J Neurosci 8:2172-2182. 
Llinas R, Walton K, Bohr V (1976) Synaptic transmission in squid giant synapse after potassium conductance blockage with external 3and 4-aminopyridine. Biophys J 16:83-86.

Llinas R, Steinberg IZ, Walton K (1981) Presynaptic calcium currents in squid giant synapse. Biophys J 33:289-322.

Llinas R, Sugimori M, Simon SM (1982) Transmission by presynaptic spike-like depolarization in the squid giant synapse. Proc Nat Acad Sci USA 79:2415-2419.

Marty A, Neher E (1985) Potassium channels in cultured bovine adrenal chromaffin cells. J Physiol (Lond) 367:117-141.

Miledi R, Slater CR (1966) The action of calcium on neuronal synapses in the squid. J Physiol (Lond) 184:473-498.

Miller DJ, Smith GL (1984) EGTA purity and the buffering of calcium ions in physiological solutions. Am J Physiol 246:C160-C166.

Nachsen DA, Drapeau P (1982) A buffering model for calcium-dependent neurotransmitter release. Biophys J 38:205-208.

Neher E (1986) Concentration profiles of intracellular calcium in the presence of a diffusible chelator. Exp Brain Res 14:80-96.

Neher E (1988) The influence of intracellular calcium concentration on degranulation of dialysed mast cells from rat peritoneum. J Physiol (Lond) 395:193-214.

Penner R, Neher E (1988) Secretory responses of rat peritoneal mast cells to high intracellular calcium. FEBS Lett 226:307-313.

Pethig RR, Kuhn M, Payne R, Adler EM, Chen T-H, Jaffe LF (1989) On the dissociation constants of BAPTA-type calcium buffers. Cell Calcium 10:491-498.

Pusch M, Neher E (1988) Rates of diffusional exchange between small cells and a measuring patch pipette. Pfluegers Arch 411:204-211.

Quast U, Labhardt AM, Doyle VM (1984) Stopped-flow kinetics of the interaction of the fluorescent calcium indicator Quin 2 with calcium ions. Biochem Biophys Res Commun 123:604-610.

Roberts WM, Jacobs RA, Hudspeth AJ (1990) Colocalization of ion channels involved in frequency selectivity and synaptic transmission at presynaptic active zones of hair cells. J Neurosci 10:3664-3684.

Sanchez ME, Nuno CM, Buchanan J, Augustine GJ (1990) Contractions of the squid stellate ganglion. J Exp Biol 152:369-387.

Simon SM, Llinas RR (1985) Compartmentalization of the submembrane calcium activity during calcium influx and its significance in transmitter release. Biophys J 48:485-498.
Smith GA, Hesketh RT, Metcalfe JC, Feeney J, Morris PG (1983) Intracellular calcium measurements by ${ }^{19} \mathrm{~F}$ NMR of fluorine-labeled chelators. Proc Nat Acad Sci USA 80:7178-7182.

Smith GL, Miller DJ (1985) Potentiometric measurements of stoichiometric and apparent affinity constants of EGTA for protons and divalent ions including calcium. Biochim Biophys Acta 839:287-299.

Smith PD, Liesegang GW, Berger RL, Czerlinski G, Podolsky RJ (1984) A stopped-flow investigation of calcium ion binding by ethylene glycol bis ( $\beta$-aminoethyl ether)- $N, N^{\prime}$-tetraacetic acid. Anal Biochem 143: 188-195.

Smith SJ, Augustine GJ (1988) Calcium ions, active zones and synaptic transmitter release. Trends Neurosci 11:458-464.

Speksnijder JE, Miller AL, Weisenseel MH, Chen T-H, Jaffe LF (1989) Calcium buffer injections block fucoid egg development by facilitating calcium diffusion. Proc Nat Acad Sci USA 86:6607-6611.

Strautman AF, Cork RJ, Robinson KR (1990) The distribution of free calcium in transected spinal axons and its modulation by applied electrical fields. J Neurosci 10:3564-3575.

Swandulla D, Hans M, Zipser K, Augustine GJ (1991) Role of residual calcium in transmitter release at the squid giant synapse. Biophys J 59:595a.

Thomas MV (1982) Techniques in calcium research. London: Academic.

Tsien RY (1980) New calcium indicators and buffers with high selectivity against magnesium and protons: design, synthesis and properties of prototype structures. Biochemistry 19:2396-2404.

Tsien RY (1989) Fluorescent probes of cell signaling. Annu Rev Neurosci 12:227-253.

Isien RY, Pozzan T, Rink TJ (1984) Measuring and manipulating cytosolic $\mathrm{Ca}^{2+}$ with trapped indicators. Trends Biochem Sci 9:263266.

Zucker RS, Fogelson AL (1986) Relationship between transmitter release and presynaptic calcium influx when calcium enters through discrete channels. Proc Nat Acad Sci USA 83:3032-3036.

Zucker RS, Steinhardt RA (1978) Prevention of the cortical reaction in fertilized sea urchin eggs by injection of calcium-chelating ligands. Biochim Biophys Acta 541:459-466. 